\title{
COVID-US: a simplified cardiopulmonary ultrasound approach to use in suspected and confirmed COVID-19
}

\author{
Nutri Virtual Symposium 2020 \\ Nutrition Battling on Pandemic COVID-19: How to Survive
}

Link to DOI: 10.25220/WNJ.V04.S3.0010

Journal Website: www.worldnutrijournal.org

Marek Nalos ${ }^{1}$

${ }^{1}$ Medical Faculty Hospital Plzen, Charles University, Czech Republic; Nepean Hospital, University of Sydney, Australia

SARS-CoV-2, a novel coronavirus, is spreading around the world, causing unprecedented morbidity and excess mortality. The characteristic feature of the COVID-19 disease is hypoxemic respiratory failure caused by viral pneumonitis, frequently accompanied by thrombosis in the pulmonary vasculature. Cardiac complications are also common and contribute to hemodynamic instability, organ failure and mortality. Using ultrasonography to help diagnose, risk stratify and treat patients suffering from COVID-19 disease is therefore intuitive and imperative.

Lung ultrasound is more sensitive than chest radiography in detecting viral pneumonitis and may be used instead of computed tomography, because it is portable, easily repeatable and does not expose patients to radiation. Indeed, studies have documented the utility of diagnosing lung consolidation, interstitial syndrome, pneumonitis, pleural effusions or pneumothorax in COVID-19 patients. Lung ultrasound findings of dorsal basal consolidation may help select patients that are likely to benefit from ventilation in prone position.

Ultrasound can also guide procedures form pleural drainage of effusions to vascular access placement.

Similarly, echocardiography is useful for cardiac conditions associated with COVID-19. Myocardial infarction, viral myocarditis, right ventricular failure, pulmonary embolism or underlying valvular or hypertensive heart disease can all be evaluated and monitored during the course of illness and allow for timely treatment.

An important aspect in the care of COVID-19 patients is to ensure that staff and other patients are protected from viral spread. Using a portable ultrasound machine avoids patient transport for CT scans thus minimising infection risks associated with transporting SARSCov2 positive patients. Staff performing ultrasound must wear adequate personal protective equipment including impermeable gown, hat, gloves, goggles and FFP2/FFP3 mask. The ultrasound machine used needs to be thoroughly decontaminated with alcohol-based solution and detergent wipes for the probes. A machine dedicated solely for COVID-19 patients is desirable.

Keywords: lung ultrasound, echocardiography, COVID-19, viral pneumonitis 
World Nutrition Journal | eISSN 2580-7013

\section{ABSTRACT}

Corresponding author:

Assoc/Prof. Marek Nalos, PhD

Medical Faculty Hospital Plzen, Charles University, Czech Republic

Nepean Hospital, University of Sydney, Australia

E-mail address: mareknalos@gmail.com 